\title{
Action-Specific Influences on Distance Perception: A Role for Motor Simulation
}

\author{
Jessica K. Witt \\ Purdue University
}

\author{
Dennis R. Proffitt \\ University of Virginia
}

\begin{abstract}
Perception is influenced by the perceiver's ability to perform intended actions. For example, when people intend to reach with a tool to targets that are just beyond arm's reach, the targets look closer than when they intend to reach without the tool (J. K. Witt, D. R. Proffitt, \& W. Epstein, 2005). This is one of several examples demonstrating that behavioral potential affects perception. However, the action-specific processes that are involved in relating the person's abilities to perception have yet to be explored. Four experiments are presented that implicate motor simulation as a mediator of these effects. When a perceiver intends to perform an action, the perceiver runs a motor simulation of that action. The perceiver's ability to perform the action, as determined by the outcome of the simulation, influences perceived distance.
\end{abstract}

Keywords: distance perception, motor simulation, affordances, perception-action coupling, intention

We perceive the world in terms of our abilities to act on it. For example, people who throw heavy balls to targets perceive the targets to be farther away than do people who throw light balls to them (Witt, Proffitt, \& Epstein, 2004). Hills look steeper when wearing a heavy backpack (Bhalla \& Proffitt, 1999). Softballs look bigger to the batter who is hitting well (Witt \& Proffitt, 2005). And, of specific relevance to the current studies, objects that are within reach as a result of holding a tool look closer than when the tool is not held and the objects are beyond reach (Witt, Proffitt, \& Epstein, 2005). These results are striking because they demonstrate that, in perception, optical and ocular-motor information are scaled to action-specific aspects of the perceiver. Fajen (2005) has also proposed that spatial perceptions are scaled by action-specific constraints. These findings motivate a search for the underlying processes that relate people's behavioral abilities to the visual information specifying environmental layout. Here, we propose that one such process is an internal motor simulation. Essentially, people imagine the performance of an intended action-either covertly or explicitly - and the outcome of this simulation influences perception.

\section{Physiological Effort and Ability Influence Perception}

Perception is, of course, primarily a function of the optical information that specifies the environment. Recent research, however, has shown that in order to optimize effective action planning, this information is scaled in perception to the perceiver's ability to perform intended actions (for reviews, see Fajen, 2005; Proffitt, in press). People see the world in a way that relates environmental affordances to their abilities to act in the world. Moreover, the

Jessica K. Witt, Department of Psychological Sciences, Purdue University; Dennis R. Proffitt, Department of Psychology, University of Virginia.

Correspondence concerning this article should be addressed to Jessica K. Witt, Purdue University, Department of Psychological Sciences, 703 Third Street, West Lafayette, IN 47907. E-mail: Jkwitt@purdue.edu specific behavioral abilities that matter depend upon what action the individual intends to perform (Witt et al., 2004, 2005). To date, there is evidence suggesting that perceived slant, distance, and size are influenced by a person's action repertoire, performance capabilities, and physiological state.

People's perception of surface layout is influenced by whether or not an intended action is possible. For example, hills that can be traversed look shallower than hills that are too steep to traverse (Proffitt, Bhalla, Gossweiler, \& Midgett, 1995). Participants estimated the steepness of hills from both the top and bottom. Estimates were approximately the same from both vantage points for hills less than about $25^{\circ}$. At and beyond this angle, however, hills appeared steeper from the top than from the bottom. For the grass-covered slopes that were used in this study, $25^{\circ}$ is approximately the angle at which it is still possible to ascend these hills, but at which it is biomechanically much more difficult to descend without falling or breaking into a run that would be difficult or impossible to stop. In other words, a $25^{\circ}$ hill affords walking for a person at the bottom of the hill; but it does not afford walking if the person is at the top. The hill looks steeper from the vantage point where walking is no longer possible than from the vantage point where walking is possible.

Perceived distance is also influenced by whether or not an action is possible. For example, reachable targets look closer than targets that are beyond reach (Witt et al., 2005). Participants estimated the distance and reached to targets that were beyond arm's reach. However, during half the trials, participants held and reached with a tool, a conductor's baton, that extended far enough so that the participants were able to touch all of the targets. Therefore, the same targets were beyond reach when participants did not hold the tool and within reach when the tool was held. Even though these targets were the same distance away, they looked closer when they were within reach because the tool was used than they did when they were beyond reach because the tool was not used.

In addition, people's performance on a given task also influences perception. For example, softball players who were hitting 
better judged the ball to be bigger than players who had more difficulty hitting (Witt \& Proffitt, 2005). After softball games, players viewed an array of varying-sized circles and selected the one they thought best matched the size of the softball. They also reported their batting average for the just played-game or games. Batting average was positively correlated with apparent ball size. Players with a higher batting average selected a larger circle as matching the size of the softball. In other words, the ball was recalled as looking bigger to players who were hitting well. A similar finding was observed in dart throwers (Wesp, Cichello, Gracia, \& Davis, 2004). Participants dropped darts onto targets, and those who were able to hit the target with fewer throws perceived the target to be bigger — as assessed via visual matching with the target present- than did participants who had more trouble hitting the target. Recently, we demonstrated that golfers judge the size of the hole to be bigger when they are playing well (Witt, Linkenauger, Bakdash, \& Proffitt, in press). Apparent hole size was negatively correlated with score on the course that day; that is, the hole looked larger to players who played better, lower scores being better in golf. These results demonstrate that perceived size is correlated with people's ability to perform a task for which size has performance relevance.

The energetic requirements to perform an action also influence perception. Both perceived slant and perceived distance were influenced by manipulations that increased the anticipated effort required to walk or throw over the relevant surface. For example, hills looked steeper to participants who were wearing a heavy backpack, fatigued after a long run, of low physical fitness, or elderly and in declining health (Bhalla \& Proffitt, 1999). Perceived slant increased as the anticipated effort required to ascend the hill increased. Similarly, distances looked farther for participants who wore a heavy backpack compared with those who wore no backpack (Proffitt, Stefanucci, Banton, \& Epstein, 2003), for participants who threw a heavy ball compared with those who threw a light ball (Witt et al., 2004), and when participants viewed targets uphill compared with on flat ground (Stefanucci, Proffitt, Banton, \& Epstein, 2005). As the anticipated effort required to walk or to throw to a target increased, perceived distance also increased.

It is not the case, however, that ability and effort always influence perception. Rather, the effects of ability and energetic costs on perception are specific to the action that people intend to perform. People see the world relative to their goals and intentions; only the behavioral ability applicable to performing an intended action influences perception. Targets within reach looked closer only when participants intended to reach to them (Witt et al., 2005). When participants never intended to reach to targets, the perceived distance to targets was unaffected by whether or not a tool was held. Similarly, only the effort associated with performing an intended action influences perception (Witt et al., 2004). After throwing a heavy ball, targets looked farther away to participants who intended to throw again but not to participants who intended to walk the extent. Likewise, experimental manipulations of anticipated effort for walking influenced perceived distance for participants who intended to walk but not for those who intended to throw a ball across the extent to a target. These experiments demonstrate that it is not people's overall potential for action that influences perception; rather, perception is scaled by their behavioral potential to perform a specific, intended action.
In summary, perception relates optical information specifying the environment to the abilities and intentions of the perceiver. People see the world in a way that is specific to their goals and to their behavioral potential to achieve these goals.

\section{Motor Simulation as the Proposed Mediator of Action- Specific Influences on Perception}

Thus far, there have been several demonstrations that people's abilities and intentions to act influence perception. However, the underlying processes that are responsible for this effect have not yet been explored. Candidate mechanisms must satisfy the following requirements: First, the process must provide an assessment of the person's ability to perform an intended action (Witt et al., 2005). Second, it should have access to both the anticipated outcome of the action as well as the energetic costs associated with the action because both of these aspects influence perception (Proffitt, 2006). Third, the process must be future oriented because people perceive the world in terms of the actions they intend to perform, not those actions they just performed (Witt et al., 2004). Finally, the process should be sensitive to the person's physical limitations (Fajen, 2005).

Internal motor simulation meets these requirements. For the purposes of this article, a motor simulation is the imagining of an action, either covertly or explicitly, without necessarily executing the action. According to Jeannerod, a motor simulation is "a representation of the future, which includes the goal of the action, the means to reach it, and its consequences on the organism and the external world" (Jeannerod, 2001, p. S103). Thus, motor simulations are future oriented and have access to the outcome of anticipated actions. Furthermore, there is behavioral and neurological evidence that suggests that motor simulation is constrained by the limitations of the body (see below).

Some of the most compelling behavioral evidence for motor simulation comes from research on mental rotation of hands (Parsons, 1987a, 1987b). The task is to judge whether a picture of a rotated hand is of a left or a right hand. In typical mental rotation tasks, the time to make a judgment corresponds with the angle of rotation (Shepard \& Metzler, 1971). Interestingly, when the task involves a body part, judgment time corresponds with the time to move one's own body part to the depicted orientation ((Parsons, 1987a, 1987b). For example, participants took longer to judge that a right hand rotated $120^{\circ}$ clockwise was a right hand than that a left hand rotated $120^{\circ}$ clockwise was a left hand because they only had to simulate rotating their left hands $120^{\circ}$ while biomechanical constraints forced them to rotate their right hands $240^{\circ}$, which took longer (Parsons, 1987b). In addition, reaction times increased when the starting hand position was at an impossible orientation, thereby delaying the ability to simulate a hand rotation and forcing participants to rely on additional processes (Petit, Pegna, Mayer, \& Hauert, 2003).

More direct support for the use of motor simulation during mental rotation of hands comes from interference paradigms. Judgment times were affected when participants had to physically rotate their own hands while performing the mental rotation task. Reaction times increased when the physical movement was in the direction opposite to the simulated movement, and they decreased when the physical movement corresponded with the direction of mental rotation (Wohlschläger \& Wohlschläger, 1998). Interfer- 
ence occurred because many of the same processes are used to simulate and execute actions. Engaging the process by simultaneously rotating one's own hand interfered with participants' ability to mentally rotate their hands.

Motor simulations have also been implicated in tasks that require making judgments about the ease of an action. Participants took longer to judge that a difficult grasping task was difficult than that an easy grasping task was easy (Frak, Paulingnan, \& Jeannerod, 2001). The authors suggested that participants had to simulate grasping the object in order to make a judgment and that judgments of difficult tasks took longer because simulating a difficult task took longer than simulating an easy task.

Evidence also suggests that people recruit motor simulation processes when explicitly imagining an action, as indicated by the parallel characteristics found between imagining and executing actions. For example, the amount of time it takes to explicitly imagine performing an action is consistent with the amount of time it would take to execute the action. Participants took just as long to physically walk to a target as they did to imagine walking to the target (Decety, Jeannerod, \& Prablanc, 1989; Papaxanthis, Pozzo, Skoura, \& Schieppati, 2002). Similarly, the time it takes to imagine reaching for a target is the same as the time it takes to actually reach for the target (Papaxanthis, Schieppati, Gentili, \& Pozzo, 2002). The time to reach and imagine reaching continued to be the same even after weight was added to the participants' hands. Corresponding durations between imagined and executed actions have been found for other tasks such as writing sentences (Decety \& Michel, 1989; Papaxanthis, Pozzo, et al., 2002), drawing figures (Decety \& Michel, 1989), and pointing to targets (Maruff \& Velakoulis, 2000).

Another similarity between imagined and executed actions is that they both conform to Fitts' (1954) Law. Just as participants were faster to point to large targets, they were also faster to imagine pointing to large targets compared with smaller targets (Maruff \& Velakoulis, 2000; Sirigu et al., 1996). Imagined times to trace figures of small and large amplitudes corresponded to the physical times to trace the figures (Decety, 1993; Decety \& Michel, 1989). Also, both executed and imagined walking times increased when the path width was narrow (Decety \& Jeannerod, 1995).

Furthermore, imagined and executed actions activate peripheral systems to similar degrees. Heart rate and pulmonary ventilation increased during imagined actions, such as walking on a treadmill (Decety, Jeannerod, Durozard, \& Baverel, 1993; Decety, Jeannerod, Germain, \& Pastene, 1991; Wuyam et al., 1995). Furthermore, this increase was proportional to the amount of effort required to perform the action (Decety et al., 1993, 1991). Heart rate increased more when participants imagined walking with a heavier load or at an increased speed.

Motor simulations are also implicated in tasks that require predicting the outcomes of observed actions. Many researchers propose that people run a motor simulation when they observe someone else performing an action (e.g., Decety \& Grezes, 1999), and Knoblich and colleagues further demonstrated that simulation produces an output that allows people to predict the outcome of an action (Knoblich \& Flach, 2001). In one experiment, participants watched videos of themselves and others throwing darts to the top, middle, or bottom portion of a target. The video only showed the throw itself, and participants had to guess where each dart would land. People were good at predicting where their own darts would land; accuracy was around $80 \%$ when participants viewed videos of their entire bodies while making their throws. Moreover, people were better at predicting where their own darts would land than where other participants' darts would land. Knoblich and Flach (2001) argued that people are better at predicting the outcome of their own actions because viewing the action engages an appropriate motor simulation that more closely matches the viewed action when viewing themselves than when viewing someone else. Thus, the outcome is more likely to be correct.

A similar paradigm was used with handwriting movements (Knoblich, Seigerschmidt, Flach, \& Prinz, 2002). Participants' movements were recorded while they wrote the number " 2 ." This movement required two strokes. One stroke started at the top left and curved down to the bottom left. The second stroke started at the bottom left and moved horizontally to the right. Movements were recorded when the two strokes were performed sequentially and in isolation of each other. One week later, participants viewed displays with a moving dot that replicated the movement of the pen when they either drew the " 2 " all at once or just the first stroke of the "2" in isolation. Participants saw the dot move along the path of the first stroke and then had to predict whether a second stroke would follow or if the first stroke had been performed in isolation. Judgments were better when participants saw their own handwriting compared with other participants' handwriting. Knoblich et al. (2002) argued that participants simulated the observed movement; and, because the observed movement more closely matched the simulated movement when viewing their own handwriting, they were better able to simulate the next movements as well. Mental stimulation can be used to predict the outcomes of observed movements and subsequent movements as well.

Research from cognitive neuroscience provides support for a neurological realization of motor simulation. The tasks that have been used to tap into simulation include both explicit and implicit imagery. Using an explicit imagery paradigm, researchers recorded brain activations during execution and imagination of an action such as moving a joystick in a sequence of movements (Stephan et al., 1995), handwriting (Decety, Philippon, \& Ingvar, 1988), hand clenching to the pace of a metronome (Ingvar \& Philipson, 1977), tapping one's fingers to a designated sequence (Gerardin et al., 2000; Hanakawa et al., 2003; Roland, Skinhoj, Lassen, \& Larsen, 1980), or grasping objects (Decety et al., 1994). In implicit imagery paradigms, participants had to make decisions about how they would act on an object. For example, in one experiment, participants saw pictures of dowels and had to determine whether they would use an underhand or overhand grip to grasp the dowel (Johnson et al., 2002). Other implicit imagery paradigms involve judging whether a depicted hand was a left or right hand (Parsons \& Fox, 1998; Parsons et al., 1995) or judging the outcome of a pointing movement (Chaminade, Meary, Orliaguet, \& Decety, 2001). These experiments provide converging lines of evidence that implicate several motor-related areas, such as the premotor cortex, supplementary motor cortex, cerebellum, and parietal cortex, as being involved in motor simulation processes. Further support comes from patient studies that demonstrate that damage to the parietal cortex (Sirigu et al., 1996) and basal ganglia (Dominey, Decety, Broussolle, Chazot, \& Jeannerod, $1995)$ interferes with the ability to imagine actions although damage to motor cortex (Sirigu et al., 1995) leaves imagined actions 
intact. Thus, there is some, though not complete, overlap in the areas involved in motor execution and simulation (see Grezes \& Decety, 2001, for review).

Taken together, these studies make several points. One is that there is fairly compelling evidence that motor simulation processes do exist and are neurologically realized. The second is that motor simulations are used in a variety of different situations, all of which involve action, whether it be executing actions, imagining actions, or making judgments about actions. Thus, it is highly likely that motor simulations are actively engaged when people perceive the world with the intention to act. Third, the process could have desirable characteristics, such as its future-oriented nature, access to anticipated action outcomes, and sensitivities to biomechanical limitations. Therefore, there are reasons to believe that motor simulations may be involved in the effects of a perceiver's abilities on perception.

\section{Overview}

In the first two experiments, we examined whether perception is influenced by anticipated changes in the perceiver's ability to act. The issue under study is whether action's influence on perception is based on people's current abilities or on those abilities that will be possessed at the time of the intended action. As discussed above, motor simulations are future-oriented processes; and, thus, their influence on perception should imply future abilities.

To dissociate between current and anticipated abilities, one group of participants viewed targets without holding a tool; thus, when distance judgments were made, the targets were out of reach. However, following the distance judgment, they picked up the tool and reached with it; thus, when these participants actually performed the intended action, the targets were within reach. This anticipatory tool-use condition was compared to two other conditions, one in which participants viewed the targets without access to a tool at any time and another in which participants held and reached with a tool throughout the entire experiment. If the effects of ability on perception are constrained to the perceiver's current abilities, then the anticipatory tool group should perceive the targets to be the same distance as the no-tool group. This is because neither group could reach the target at the time that they made their distance judgments. On the other hand, motor simulation will be implicated if those who anticipate holding a tool see the targets as closer, as do those in the hold-tool condition. This is because, at the time that the intended action is made, the targets are within reach for both groups.

In the remaining experiments, we used methods that entailed interfering with the motor simulation. This method is motivated largely by the brain-imaging literature suggesting that the neural mechanisms involved in motor simulation are also involved when physically executing an action. If motor simulations are responsible for action-specific influences on perception, then performing concurrent tasks that engage the relevant motor simulation mechanisms should eliminate the action-specific effects.

Given the overlap in neural activations between executing and simulating actions, performing concurrent actions should interfere with people's ability to simulate. Indeed, behavioral evidence supports this conclusion. As discussed above, physically rotating one's own hand influences handedness judgments of depicted hands, which rely on a motor simulation of hand rotation (Wexler,
Kosslyn, \& Berthoz, 1998; Wohlschläger \& Wohlschläger, 1998). Similarly, walking interferes with perception of point-light walkers (Jacobs \& Shiffrar, 2005). Participants' judgments of the speed of the point-light walkers were impaired when participants had to walk on a treadmill while making their judgments compared with when they just stood still. Interestingly, interference is specific to the simulated task. Walking interfered with perceiving point-light walkers, but riding on a stationary bike did not show interference (Jacobs \& Shiffrar, 2005). Similarly, physical hand rotations in a different plane (Wohlschläger \& Wohlschläger, 1998) or even at different speeds (Wexler et al., 1998) did not interfere with mental hand rotation.

\section{Experiment 1: Dissociating Current and Simulated Abilities}

People's abilities change constantly. When people grasp a tool, they instantly become capable of reaching to farther targets. As we have shown previously, when people hold a tool, targets that were beyond reach without the tool but within reach with it look closer (Witt et al., 2005). What would happen if people viewed a target with a tool being out of hand but available for use? Would they perceive the target in terms of their current ability to act, which is that the target is beyond reach and, therefore, should look farther away relative to when a tool is held? Or would they perceive the target in terms of their anticipated ability to act, which is that the target will be within reach once the tool is picked up? According to our hypothesis, perception is influenced by what people are going to do next; and, thus, it should be affected by their future abilities, not their current ones. Therefore, people should perceive the target as being closer if they know that they are going to pick up the tool even if the target is beyond reach when they estimate distance.

Another way to dissociate between simulated and current abilities is to have participants imagine holding the tool when they reach. As discussed above, the processes involved in simulating an action are also used when explicitly imagining an action. Thus, reaching with an imaginary tool should produce the same outcome from a simulation, namely that the target is within reach. By dissociating between the outcome of an imaged motor simulation and the perceiver's actual capabilities at the time that they judge target distance, we can determine which ability (simulated or current) influences perception.

\section{Method}

Participants. Thirty-two students (20 women, 12 men) participated in exchange for credit in a psychology course. All were naïve to the purpose of the experiment.

Materials and Stimuli. Stimuli were presented on a large rectangular table $(183 \mathrm{~cm}$ wide $\times 122 \mathrm{~cm}$ deep) from a downward facing projector (see Figure 1). Directly in front of the participant, a small, white paper circle $(3 \mathrm{~cm}$ in diameter) was on the table. Participants used this circle as a reference point for estimating distances. There were no other marks on the table, and the surface was homogenous and provided minimal landmarks that might be used when estimating distances. Stimuli consisted of three white, projected circles, each $1 \mathrm{~cm}$ in diameter. Target distances ranged from $74 \mathrm{~cm}$ to $120 \mathrm{~cm}$. Some participants used a conductor's baton that was $39 \mathrm{~cm}$ in length. 

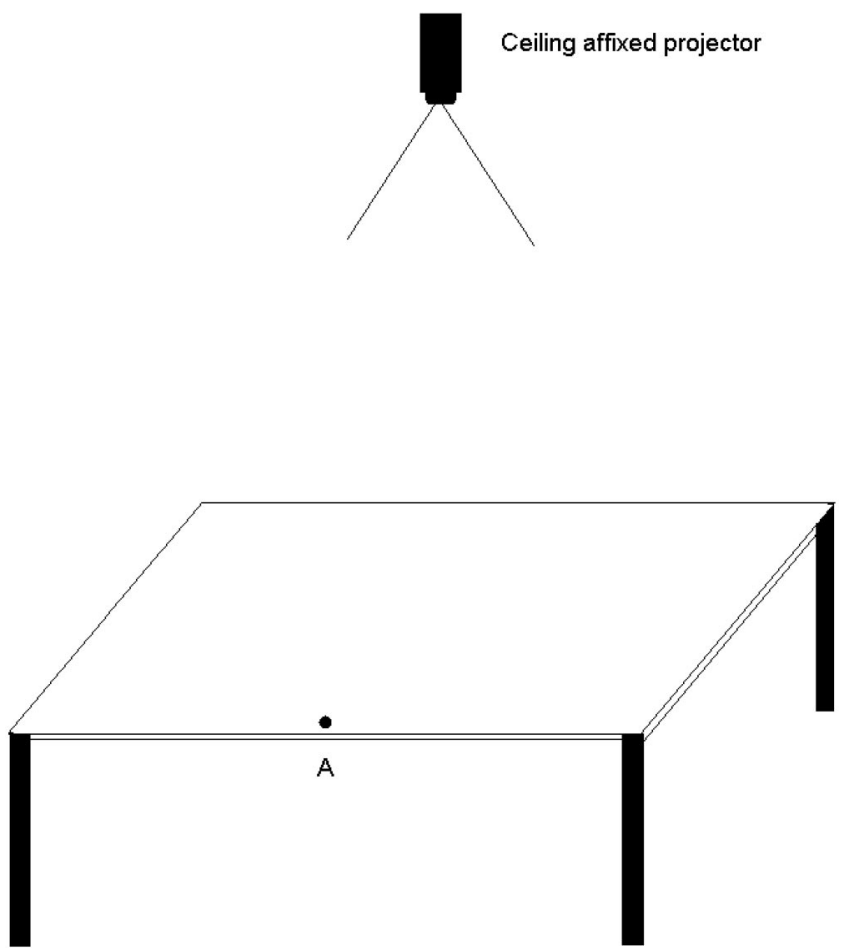

Figure 1. Experimental set up. Stimuli were projected down from a ceiling-affixed projector. Participants sat at the location marked by A. A paper circle was placed directly in front of the participants and was used in the perceptual matching task.

Design. On each trial, participants estimated the distance to a target by performing a perceptual-matching task (described below) and then reached to the location where the target had been presented. We manipulated the participants' ability to reach to the target. Participants were assigned to one of four conditions. In the No-Tool condition, participants reached with their hand, which prevented them from being able to reach most of the targets. In the Hold-Tool condition, participants held the baton throughout the entire experiment and were able to reach to all targets with it. In the Anticipate-Holding-Tool condition, the baton was lying on the table while participants made their distance judgments. Then participants picked up the baton and reached to the target. In the Imagine-Holding-Tool condition, the baton was lying on the table and participants reached to the target while imagining holding the baton in their hands. We opted to use a between-subjects design so that participants were unaware of the other conditions and thus were less likely to guess our hypothesis and adjust their distance judgments to be based on anything other than their perception of the distance to the target.

Procedure. On each trial, participants completed the perceptual-matching task and then reached to the target. The perceptual-matching task involved manipulating two comparison circles in the frontoparallel plane so that the distance between these circles was the same as the distance between the reference mark on the table in front of the participant and the target (see Figure 2). The comparison circles always started $5 \mathrm{~cm}$ on either side of the target. To move the comparison circles, participants pressed the Left- and Right-Arrow keys on a keyboard with their left hands. Pressing the Right-Arrow key made both circles move apart from each other at the same rate, and pressing the Left-Arrow key made both circles move towards each other at the rate, so the target was always directly in between the two comparison circles. The keyboard was positioned on a stand to their left. After the participants matched the distances, the target and comparison circles disappeared, and the participant reached to the location where the target had been. In the No-Tool condition, participants were instructed to reach as far as they could and then touch the target or, if it was out of reach, point to its location. In the Hold-Tool condition, participants reached to the target with the baton. In the Anticipate-Holding-Tool condition, they picked up the baton after matching the distances, touched the target location with the baton, and then put the baton back on the table before the next trial started. In the Imagine-Holding-Tool condition, participants imagined that they held the baton and touched the location of the target with the imagery end of the baton. One block of trials consisted of 10 targets ranging from $74 \mathrm{~cm}$ to $120 \mathrm{~cm}$. Participants completed two blocks of trials, and target distance within block was randomized. Perceived distance was calculated as the distance between the two comparison circles, which was digitally recorded in pixels and later transformed into centimeters. For data analyses, we took the mean for each participant for each test distance.

\section{Results and Discussion}

As can be seen in Figure 3, participants in the No-Tool condition perceived the targets to be farther away than participants in the other three conditions. We ran an analysis of variance (ANOVA) with tool condition and distance as independent factors and matched distance as the dependent factor. Distance was significant, $F(9,280)=44.84, p<.001, \dot{\eta}_{\mathrm{p}}^{2}=.59$. Tool was significant, $F(3,280)=7.20, p<.001, \dot{\eta}_{\mathrm{p}}{ }^{2}=.07$. Post hoc tests revealed that

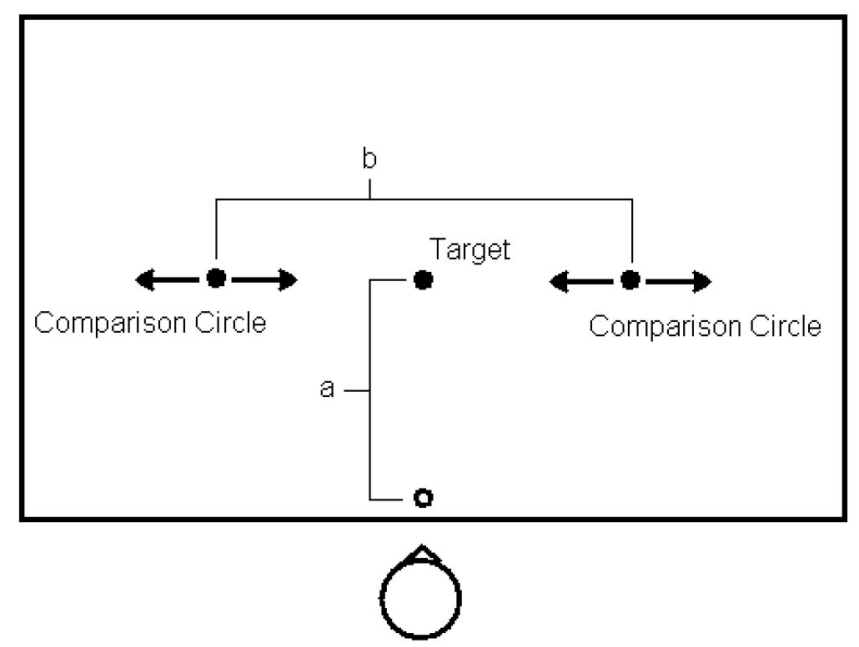

Figure 2. Perceptual matching task used in Experiments 1-3. Using their left hands, participants pressed the Left- and Right-Arrow keys on a keyboard positioned to their left. They could move the comparison circles (b) closer together or farther apart and were instructed to position the distance between the comparison circles to be the same as the egocentric distance between them (as marked by the paper circle directly in front of them) and the target (a). 


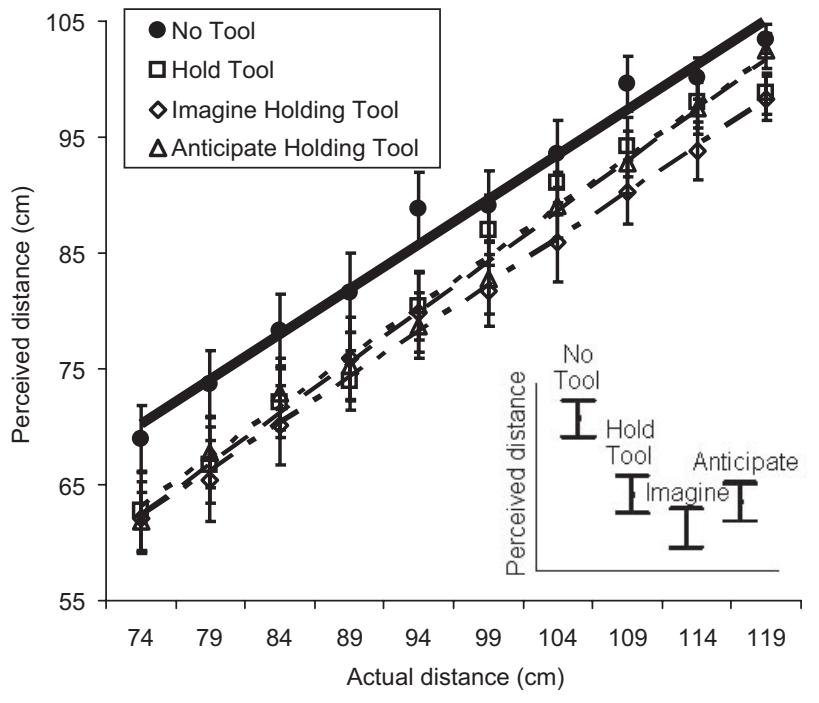

Figure 3. Perceived distance as a function of the actual distance to the target for the four conditions in Experiment 1. Error bars represent one standard error. Lines represent linear fit lines. The insert shows the main effect of condition collapsed across all target distances.

the No-Tool condition was significantly different from the three baton conditions (all $p s<.01$ ) and that the various baton conditions were not significantly different from each other (all $p$ s $>$ .69). The interaction between tool and distance was not significant, $F(27,280)=0.20, p>.99$.

We replicated our initial results (Witt et al., 2005), which demonstrated that perceived distance is compressed when targets are within reach while wielding a tool compared with when targets are beyond reach without the tool. In addition, the results show that people perceive the world in terms of their anticipated abilities to perform an action rather than their current abilities. Even though the targets were beyond reach for the Anticipate-Holding-Tool group when they made their distance estimates, this group still perceived the targets to be closer because they knew they would have the tool when they reached to the target and therefore could simulate reaching to the target with the tool. This result suggests that the outcome of the simulation, rather than the perceiver's ability at the time of estimating distance, influences perception.

It makes sense that the process of simulation allows people to simulate anticipated abilities because that may be how people know what size tool they would need to acquire in order to reach a target. When people cannot perform an action, they must be able to figure out what change in the body or environment would be necessary to complete the task. Being able to simulate using a tool that one is not currently holding allows the perceiver to test out different situations mentally rather than pick up and try to use different tools to figure out which one would get the job done.

We also found that simply imagining holding a tool influences perceived distance. Targets looked closer when people held an imaginary tool while reaching to them compared with people who just reached with their finger. So although both groups had the same physical ability to reach (or not reach) the target, the group that imagined holding a tool perceived the targets to be closer. This finding is consistent with our simulation hypothesis because the outcome of the simulation of reaching with an imagined tool is that the target is within reach, whereas the outcome of simulating reaching with no tool is that the target is beyond reach. Thus, a simulation process would relay two different outputs to perception for the two groups and thus could account for the differences between the No-Tool and Imagine-Holding-Tool conditions.

Our results are unlikely to be due to the mere presence of the tool influencing perception. In our previous article (Witt et al., 2005), participants who held the tool but did not intend to reach with it did not perceive the targets to be closer. Furthermore, participants who reached with the tool first and then reached without the tool perceived the targets to be farther away when they reached without the tool. In this case, these participants were aware of the presence of the tool because it had just been taken away from them, yet they did not perceive the targets as being closer. So the presence of a tool alone is not enough to influence perceived distance.

\section{Experiment 2: Imagining Impossible Actions}

Admittedly, it is a little strange that one can see the world in terms of imagined abilities, and our result begs the question of whether there are any limitations to this effect. Thus, we ran a second experiment where we instructed some participants to imagine an impossible action. Specifically, we asked them to imagine that their arms could extend all the way to the targets (similar to Inspector Gadget from the popular children's cartoon). According to our account of simulation, they would not be able to simulate imaging an impossible action because the processes involved in simulation are restricted by the person's physical abilities. As opposed to engaging a motor simulation, participants would have to rely on other processes, such as visual imagery, to imagine that their arms can extend to the target. Thus, if the apparent distance is influenced by a motor simulation, then the simulation will determine that the target is beyond reach, and it will look farther away. Alternatively, if imaging an impossible action can make targets look closer, then we should reconsider these effects as being a product of cognitive correction, response bias, or some other postperceptual effects.

\section{Method}

Participants. Twelve students (7 women, 5 men) participated in exchange for credit in a psychology course. All were naïve to the purpose of the experiment.

Materials and Stimuli. The materials and stimuli were the same as in Experiment 1.

Design. As in Experiment 1, participants estimated the distance to the target by performing the perceptual matching task and then reached to the target. We used the same two control conditions with one group of participants reaching to targets with their finger (No-Tool condition) and one group holding and reaching to targets with the baton (Hold-Tool condition). The critical condition in this experiment was the Imagine-Arm-Stretch condition. When these participants reached to the target, we instructed them to imagine that their arms could stretch out and extend all the way to the target. We gave them the example of Inspector Gadget. Although the participants thought the experiment was strange, they all understood the task. 
Procedure. Participants were assigned to one of the three conditions in alternating order. The procedure was the same as in Experiment 1 except that participants completed three trials at every target distance.

\section{Results and Discussion}

As can be seen in Figure 4, participants in the Imagine-ArmStretch condition perceived the targets to be just as far away as people in the No-Tool condition. An ANOVA with condition and distance as independent factors and perceived distance as the dependent factor revealed a significant effect for condition, $F(2$, $90)=6.44, p<.005, \dot{\eta}_{\mathrm{p}}{ }^{2}=.13$. Post hoc analyses showed that the Imagine-Arm-Stretch group perceived the targets to be farther away than the Hold-Tool group, $p<.001$. There was no significant difference between the No-Tool condition and the ImagineArm-Stretch condition, $p>.53$. As expected, the Hold-Tool group perceived the targets to be closer than the No-Tool group, $p<$ .001 . The effect of distance was also significant, $F(9,90)=29.61$, $p<.001, \hat{\eta}_{\mathrm{p}}^{2}=.75$. There was not a significant interaction between distance and condition, $F(18,90)=0.07, p>.99$.

This result suggests that the effects are limited to actions that are possible and therefore could be simulated using the same processes as those used to plan and execute actual movements. Furthermore, this result provides evidence that the effects of reachability on perceived distance are not due to cognitive correction because participants were told to think about the target as if it were within reach. If participants in our previous experiments (Witt et al., 2005) had perceived the targets to be the same distance away, regardless of whether the targets were within reach or not, but had reported them to be at different distances, perhaps because they surmised our hypothesis, then the participants in Experiment 2 would also have made the same corrections to their distance estimates. In other words, if participants think that they should

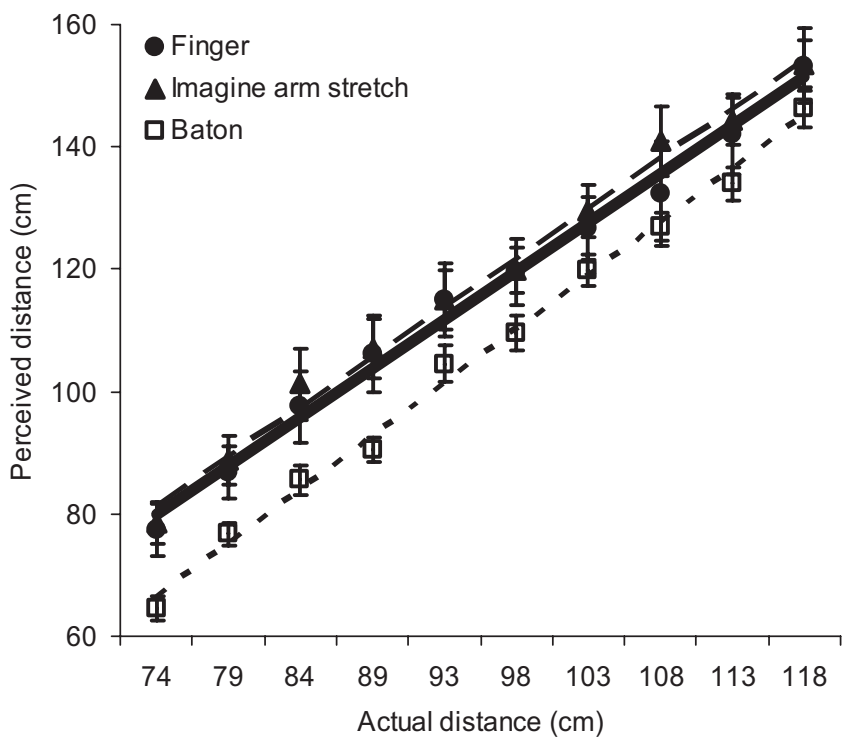

Figure 4. Perceived distance as a function of the actual distance to the target for the three conditions in Experiment 2. Error bars represent one standard error. Lines represent linear fit lines. report that targets within reach are closer, then we would have seen similar effects of reachability in Experiment 2. In contrast, even though participants were told to think of the targets as being within reach, the targets still looked farther away.

\section{Experiment 3A: Interfering with Simulation}

Although the results from the first two experiments are consistent with an account of motor simulation, they by no means prove that simulation is responsible for the effects of reachability on perceived distance. In order to obtain more direct evidence, we decided to interfere with processes of simulation directly to see if interference would eliminate the effect.

As discussed above, the processes involved in motor simulation engage many of the same neural systems as those responsible for planning and executing actions. Therefore, performing a concurrent action should interfere with simulation, so there should be no outcome of the simulation to inform perception of whether or not the target is within reach. Therefore, even if a target is within reach as a result of holding a tool, the target should only look closer for participants who are able to simulate reaching and not for participants who cannot simulate a reach.

\section{Method}

Participants. Sixteen students (10 women, 6 men) participated in exchange for credit in a psychology course. All were naïve to the purpose of the experiment.

Materials and Stimuli. The materials and stimuli were the same as in Experiment 1. In addition, a rubber ball $6 \mathrm{~cm}$ in diameter was also used.

Design. Participants estimated the distance to the target by performing the perceptual matching task and then reached to the target with the baton. The baton was lying on the table while they made their distance judgments, and then they picked up the baton prior to reaching and put the baton back on the table after completing their reach (analogous to the Anticipate-Holding-Tool condition in Experiment 1). There were two groups in this experiment. The Squeeze group squeezed a rubber ball with their right hands while making their distance judgments. They put the ball down and picked up the baton to reach, after which, they put the baton down and picked the ball back up and started squeezing before making their next estimate. The No-Squeeze group did not do anything extra with their right hand. Both groups used their left hands to manipulate the comparison circles by pressing the Left- and RightArrow keys on a keyboard positioned to their left.

Procedure. Prior to each trial, the Squeeze group applied a slight tension to the rubber ball with their right hands. On each trial, a target appeared with two comparison circles on either side. Participants estimated the distance to 10 targets ranging from 74 $\mathrm{cm}$ to $120 \mathrm{~cm}$ by manipulating the distance between the two comparison circles. After making each distance estimate, the circles disappeared. The No-Squeeze group picked up the baton and reached to the location where the target had been. The Squeeze group put the rubber ball down, picked up the baton, and reached to the target's location. Participants estimated the distance to each target twice, and target distance was randomized within block. 


\section{Results and Discussion}

As can be seen in Figure 5, participants in the Squeeze condition did not perceive the targets to be as close as participants in the No-Squeeze condition. An ANOVA with condition and distance as independent factors and perceived distance as the dependent factor revealed a significant effect for condition, $F(1,140)=13.99, p<$ $.001, \hat{\eta}_{\mathrm{p}}^{2}=.09$. The effect of distance was also significant, $F(9$, $140)=51.88, p<.001, \dot{\eta}_{\mathrm{p}}^{2}=.77$. There was not a significant interaction between distance and condition, $F(9,140)=0.93, p=$ .50

We know from Experiment 1 that people who perceive targets with the intention to pick up a tool and reach to them perceive the targets to be closer compared with people who reach with their finger. However, when participants squeezed a ball when making their distance judgments, the effect was eliminated. We propose that squeezing the ball interfered with people's ability to simulate reaching to the target with the baton. Without having the outcome of a motor simulation indicating that the target is within reach, the target should not look closer. This is what we found.

These results also suggest that the interference is effectorspecific because both groups of participants used their left hands to press buttons on the keyboard to move the comparison circles. Thus, their left hands were engaged in another task, but only concurrent actions with their right hands interfered with the simulation and the effect of reachability on perception.

We do not think that the results are due to demands on working memory or attention because participants did not pump-squeeze the ball. They simply applied a constant tension to the ball, so attentional demands were minimal. Once they applied the tension to the ball, they did not have to do anything else until it was time to reach to the target. However, to assess whether the act of

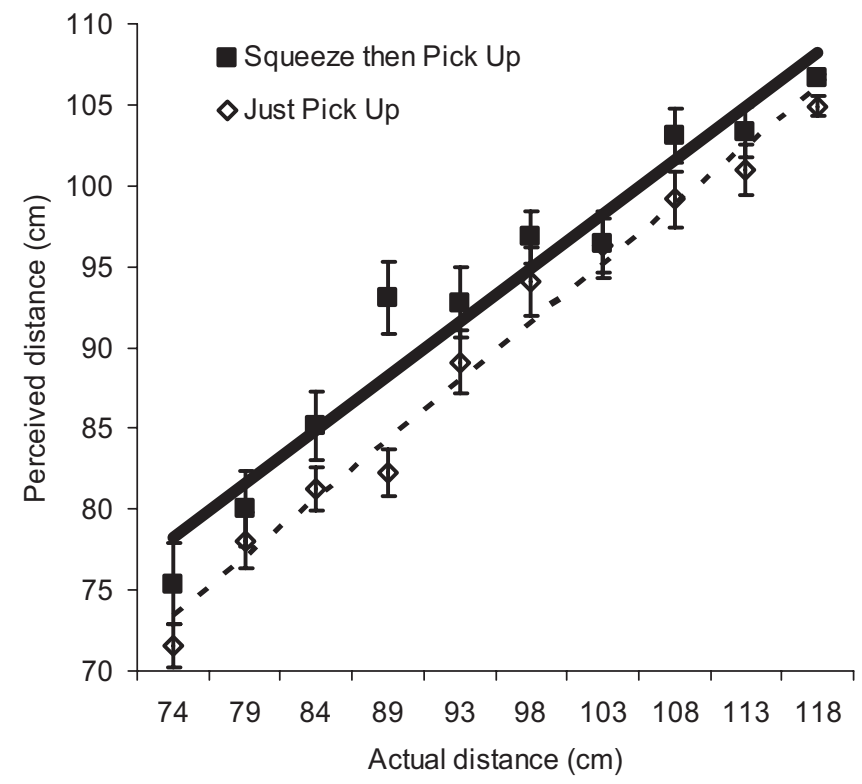

Figure 5. Perceived distance as a function of the actual distance to the target for the Squeeze and No-Squeeze conditions in Experiment 3A. All participants reached to the target with the tool. Error bars represent one standard error. Lines represent linear fit lines.

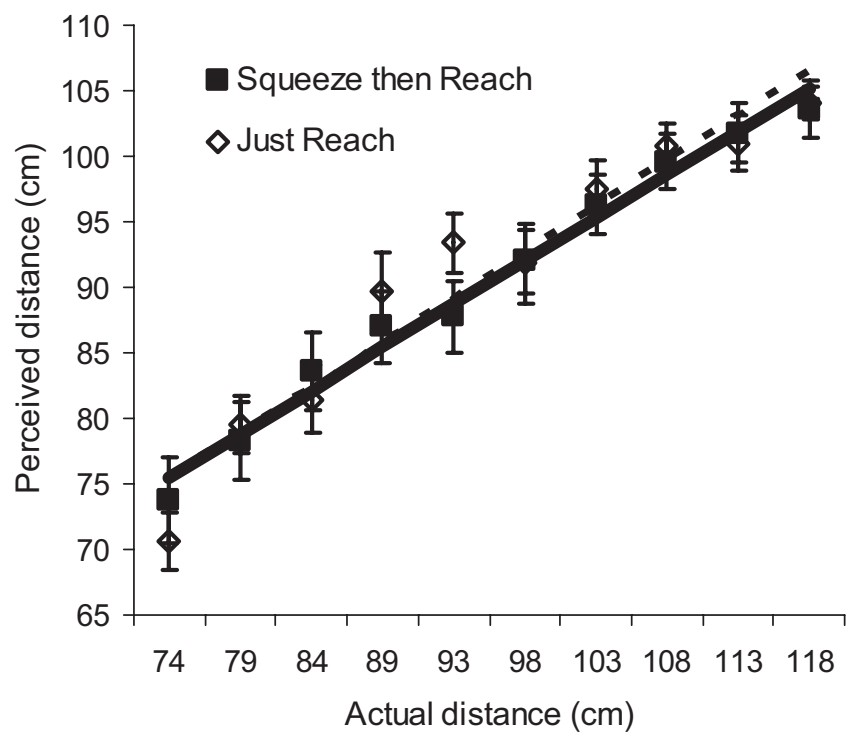

Figure 6. Perceived distance as a function of the actual distance to the target for the Squeeze and No-Squeeze conditions in Experiment 3B. Participants reached to the targets without the tool. Error bars represent one standard error. Lines represent linear fit lines.

squeezing the ball had an influence on apparent distance independent of interfering with simulating tool use, we ran a follow-up study using the ball squeeze manipulation but having participants reach without the tool. We would not expect any differences between the two groups, but if the ball squeezing task affected perceived distance independently of its effects of interfering with motor simulation, then we would find a difference in the no-tool experiment as well.

\section{Experiment 3B: Interference Without a Tool}

In order to ensure that squeezing a ball did not interfere with something other than simulating reaching with a baton, we ran a control experiment where people estimated distances and reached to targets without the tool. One group of participants squeezed a ball while making their distance judgments.

\section{Method}

Participants. Sixteen students ( 9 women, 7 men) participated in exchange for credit in a psychology course. All were naïve to the purpose of the experiment.

Materials and Stimuli. The materials and stimuli were the same as in Experiment 3A except that the baton was not used.

Design and Procedure. The design and procedure were the same as in Experiment 3A except that participants reached with their fingers instead of with the baton.

\section{Results and Discussion}

As can be seen in Figure 6, participants in the Squeeze condition perceived the targets to be just as far as participants in the NoSqueeze condition. An ANOVA with condition and distance as independent factors and perceived distance as the dependent factor 
revealed only a significant effect for distance, $F(9,140)=20.29$, $p<.001, \eta_{\mathrm{p}}^{2}=.57$. The effect of condition was not significant, $F(1,140)=0.20, p=.66$. There was not a significant interaction between distance and condition, $F(9,140)=0.28, p=.98$. The act of squeezing a ball did not influence perceived distance. Thus, the results from Experiment $3 \mathrm{~A}$ are likely to be due to the interference caused by squeezing a ball on simulating reaching with the tool rather than to something inherent to squeezing a ball.

\section{Experiment 4: Generalizing Simulation to Effort}

The results of Experiments $3 \mathrm{~A}$ and $3 \mathrm{~B}$ provide strong support of a simulation account being responsible for the effect of reachability on perceived distance. It is hard to imagine another account of these effects that could explain why simply applying tension to a rubber ball while making distance judgments prior to reaching with a tool would have an impact on distance perception. In the final experiment, we tested whether this account generalizes to the effect of effort on perception. In previous research, we demonstrated that effort for throwing influences perceived distance (Witt et al., 2004). Participants who threw a heavy ball perceived targets to be farther than participants who threw a light ball. Using the same ball squeeze manipulation, we tested if simulation was responsible for the effects of effort on perception as well.

\section{Method}

Participants. Ten women ${ }^{1}$ participated in exchange for credit in a psychology course. All were naïve to the purpose of the experiment.

Materials and Stimuli. Participants made distance judgments in a flat, grassy field. Golf tees were used to mark distances ranging from 3 to $11 \mathrm{~m}$ from the observer. The tees were placed flush with the ground so that participants could not see them. Four rows of tees were arranged in a radial pattern with the observer being located at the center. The tees facilitated the placement of a small orange disc cone used to mark each target distance. Practice distances were placed at 3,5,7, and $9 \mathrm{~m}$. Test targets were placed at $4,6,8$, and $10 \mathrm{~m}$. The target cone was placed on a different radius each time, so participants could not use previous estimates to aid in follow-up estimates. Participants threw a medicine ball $(19 \mathrm{~cm}$ in diameter) that weighed $0.91 \mathrm{~kg}$. Participants in the Squeeze conditioned used the same rubber ball as in Experiment 3.

Design and Procedure. Participants were assigned to the Squeeze condition or the No-Squeeze condition. They completed one practice block $(3,5,7$, and $9 \mathrm{~m})$ and one test block $(4,6,8$, and $10 \mathrm{~m}$ ). During the practice block, both groups threw to each target three times regardless of whether they hit the target. Then they verbally estimated the distance to the target in feet. Distance order was randomized, and each distance was presented on a different radius.

During test, both groups estimated the distance to the target first and then threw the heavy ball to the target. Both groups faced the other direction while the target was being set-up. The Squeeze group was told to start squeezing the ball before turning to face the target. Once the target was in place, participants turned towards the target and estimated the distance to the target. The Squeeze group squeezed the rubber ball the entire time that they viewed the target while estimating its distance. After making each distance judg-

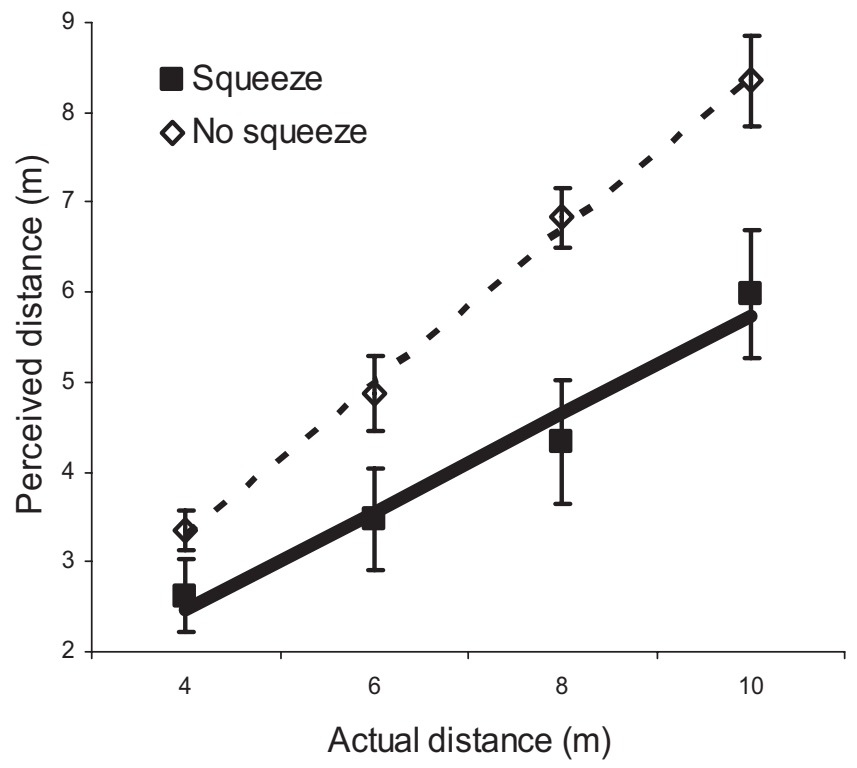

Figure 7. Perceived distance as a function of the actual distance to the target for the Squeeze and No-Squeeze conditions in Experiment 4. All participants threw the heavy ball. Error bars represent one standard error. Lines represent linear fit lines.

ment, the Squeeze group stopped squeezing the rubber ball and either put it on the ground or put it in their pocket. Neither group held the heavy ball when making their distance judgments. After each judgment, the experimenter threw the heavy ball to the participant. Then both groups threw the heavy ball to the target until they hit it or for a maximum of three throws. Distance order was randomized, and each of the four test distances was presented on a different radius.

\section{Results and Discussion}

As can be seen in Figure 7, participants in the Squeeze condition perceived the targets to be closer than participants in the No-Squeeze condition. An ANOVA with condition and distance as independent factors and perceived distance as the dependent factor revealed a significant effect for condition, $F(1,32)=23.89, p<.001, \dot{\eta}_{\mathrm{p}}{ }^{2}=.43$. The effect of distance was also significant, $F(3,32)=25.26, p<$ .001, $\eta_{\mathrm{p}}^{2}=.70$. There was not a significant interaction between distance and condition, $F(3,32)=1.37, p=.27$.

It should be noted that distances beyond $2 \mathrm{~m}$ are typically underestimated (e.g., Loomis, Da Silva, Fujita, \& Fukusima, 1992; Philbeck \& Loomis, 1997). So it is not that the No-Squeeze group was more accurate but rather that the No-Squeeze group perceived the targets to be farther away than the Squeeze group because the No-Squeeze group was able to simulate throwing the heavy ball and thus perceived distance expanded.

Consistent with our findings in Experiment 3A, interfering with the ability to simulate the intended action eliminates the effect. In this experiment, the initial effect was that throwing a heavy ball makes the targets look farther away. However, when participants

\footnotetext{
${ }^{1}$ We only ran women because only women signed up for the study.
} 
could not simulate throwing the heavy ball, the targets did not look farther away and hence looked closer compared with the group that could simulate throwing. In Experiment $3 \mathrm{~A}$, the initial effect was that targets looked closer when reaching with a tool, so interfering with the ability to simulate reaching eliminated the effect of the targets' looking closer, so the targets looked farther away compared with participants who could simulate reaching. In other words, even though the effects in Experiment $3 \mathrm{~A}$ and 4 go in different directions, the basic finding is the same. Performing an appropriate concurrent action, which interferes with the ability to simulate an intended action, eliminates the action-specific effects of effort or ability on perception.

Although Experiment 3B suggests that squeezing a ball does not influence distance perception itself, we ran a small control study with the ball-squeeze manipulation and throwing a lighter ball, which takes much less effort to throw. The ball was slightly larger than the medicine ball $(23 \mathrm{~cm}$ in diameter) but weighed only .35 $\mathrm{kg}$. We ran 8 participants using the same design as Experiment 4 and found no difference between the Squeeze (mean $=5.30 \mathrm{~m}$, $S D=1.93)$ and No-Squeeze $($ mean $=5.05 \mathrm{~m}, S D=2.77)$ conditions, $F(1,24)=0.16, p=.69$. There was a significant effect for distance, $F(3,24)=10.86, p<.001, \dot{\eta}_{\mathrm{p}}{ }^{2}=.58$. The interaction between condition and distance was not significant, $F(3$, $24)=0.11, p=.94$. Thus, the ball-squeeze effect observed with the heavy ball is likely due to interference with participants' ability to simulate throwing the heavy ball and its effect on perception.

These results suggest that motor simulation processes are involved in the effects of behavioral abilities and effort on distance perception. In addition, our account of simulation generalizes to perception in different spaces, namely near space and far space.

\section{General Discussion}

There are now several demonstrations that a person's ability to act influences perception. Distances appear farther with increased effort to walk (Proffitt et al., 2003; Stefanucci et al., 2005) or to throw (Witt et al., 2004). Hills appear steeper with increased walking effort (Bhalla \& Proffitt, 1999; Proffitt et al., 1995). Targets within reach look closer than targets beyond reach (Witt et al., 2005). Perceived size is influenced by the perceiver's ability to hit the target (Wesp et al., 2004; Witt et al., in press; Witt \& Proffitt, 2005).

In this article, we propose that when perceivers view an environment with the intention to perform an action, they run an internal motor simulation of the action and that the outcome of this simulation influences perception. As described in the introduction, the simulation is a type of first-person motor imagery, which can be unconscious. We tested our hypothesis of simulation in two ways. First we dissociated between the perceiver's current and anticipated ability to act. Participants estimated the distance to targets that were beyond reach of their hands but within reach if they held a tool. When they made their distance judgments, they were not holding the tool, so the targets were beyond reach. However, participants knew that they would be able to pick up and reach with a tool, so we hypothesized that they would simulate reaching with the tool. The outcome of simulating a reach with a tool would be that the target was within reach even though the target was physically beyond reach at the time that the estimate was made. We found that targets looked closer to participants who anticipated being able to reach to the targets with the tool compared with participants who did not use the tool.

We also found that targets looked closer when participants reached to them with an imaginary tool. In this situation, participants should still simulate reaching with a tool, so the outcome of the simulation should be that the target was within reach even though the target was never physically within reach. The processes underlying simulation are also recruited when explicitly imagining an action (see the introduction). Thus, these processes will be engaged when simulating an anticipated action and when simulating an imagined action. When the outcome of a simulation was that the target was within reach, the target looked closer even though participants only imagined holding a tool.

However, when there was no outcome of the simulation indicating that the target was within reach, then the target did not look closer. In Experiment 2, participants imagined a physically impossible action, namely that their arm could stretch beyond its physical limits. Simulation is limited by the actual biomechanical constraints of the body, so participants were not able to simulate the impossible action of stretching out their arms and had to rely on other processes, such as visual imagery, to perform the task. Even though participants were told to imagine that the targets were within reach, they did not perceive the targets to be closer.

We hypothesized that the participants in Experiment 2 could not simulate reaching to the targets because they could not simulate an impossible action, and, because there was no outcome of the simulation indicating that the targets were within reach, the targets did not look closer. Experiment 2 raises several questions about the nature of simulation and what kinds of actions can be simulated. Impossible actions are generally of three sorts. One is that the task is impossible for an individual person even if that action could be possible for another person. For example, the second author cannot perform the splits. Splits are not an impossible action for everyone, but they are impossible for him. Although unlikely, the second author might be able to perform a split with practice. Other actions, however, are impossible for everyone and will always be so, such as stretching one's arm out $2 \mathrm{ft}$ beyond its limit. A third way in which actions may be impossible is that an action that once was possible may no longer be possible. For example, people who have amputated limbs can no longer reach the way they could before their limb was amputated. In summary, there are actions that are not possible but could be possible, actions that will never be possible, and actions that were once possible but are no longer so. It would be interesting but beyond the scope of this article to explore all types of imagined actions to determine which ones do and do not influence perception. However, we have begun to set up the boundaries of the effect. Experiment 1 showed that imagining a simple and immediately possible action did influence perception, whereas Experiment 2 showed that imagining an action that has always been and will always be impossible did not influence perception.

The second way that we tested our hypothesis that simulation processes are involved in the effects of reachability on perception was to use an interference paradigm. Given the research suggesting neurological and behavioral overlap between simulating and executing actions (see the introduction), performing a concurrent action with the acting hand when estimating distance should interfere with the simulation. If there is no outcome from the simulation to inform perception of the perceiver's abilities to act, 
then any effects of ability on perception should be eliminated and people should perceive the distance to the targets without influence from the intended action.

We demonstrated that interfering with the simulation eliminated the effects of ability on perception in two different contexts. In Experiment 3A, participants reached to targets with a tool, so the targets should have looked closer. However, one group of participants squeezed a rubber ball while making their distance judgments. Thus, they could not simulate that the targets were within reach. This eliminated the effect of reachability on perception, so the targets looked farther away to these participants than to participants in the No-Squeeze condition who reached with a tool and could simulate reaching. Because both groups of participants performed a concurrent action with their left hands, namely pressing the Left- and Right-Arrow keys in order to move the comparison circles, it is likely that the effects of interference are specific to the effector used to perform the intended action.

Performing a concurrent action also interfered with the effect of effort for throwing on perceived distance. Targets look farther away to people who throw a heavy ball compared with people who throw a light ball (Witt et al., 2004). However, if the throwers cannot simulate throwing the heavy ball, then even though it takes more effort to throw the heavy ball to the target, it should not look farther away because there is no outcome from the simulation to inform perception that the action will take more effort. In Experiment 4, targets looked farther away to participants in the NoSqueeze condition who threw the heavy ball and could simulate throwing than to participants in the Squeeze condition who threw the heavy ball but could not simulate throwing. When participants could not simulate the action, the intended action had no influence on perception, but when they could simulate the intended action, perception was influenced by the anticipated effort associated with throwing the heavy ball.

Applying a constant tension to the rubber ball required minimal attention and minimal demands on working memory. Control experiments demonstrated that squeezing the ball itself did not influence distance perception. Furthermore, participants who squeezed the ball perceived the targets as being farther away in Experiment 3A but closer in Experiment 4. We do not think an account of working memory can explain this pattern of results. In contrast, our account of motor simulation can explain how the simple act of squeezing the ball altered the way the world looked for participants whose abilities and energetic costs were manipulated. Squeezing the ball interfered with motor simulation, so when the targets should have looked closer because they were within reach (as in Experiment 3A), they instead looked farther away compared with a group who could simulate reaching. Similarly, when the targets should have looked farther away because they required more effort to throw the heavy ball (as in Experiment 4), they looked closer compared with a group that could simulate throwing the heavy ball. An account of perception that is consistent with these findings is that perceivers simulate their intended actions and the outcome of the simulation serves to scale perception.

\section{Implications for Theories of Simulation}

Exploring our environments is an essential ability that our actions and our perception allows us to do. Simulation affords a different kind of exploration that is also very useful. Through simulation, people can examine different possibilities for action. They can try several actions before committing to performing a specific one. If an action is not possible given their current bodies, people can simulate using various tools in order to determine which tool to acquire in order to achieve their goals. People can also determine which route to take or what speed to move at based on simulations of different routes or speeds. Simulation is useful for determining and planning the next action and promotes a sort of exploration that entails minimal costs and minimal risks. Little energy is wasted simulating various options compared with the energetic costs of trying all possible actions before deciding which was best. Prior simulation also helps prevent people from performing actions that might be dangerous because, if the outcome of the simulation suggests that they will fall, experience a collision, or otherwise be put in harm's way, then they will likely find an alternative course of action.

Accounts of simulation are pervasive in a variety of situations. For example, simulation is argued to be used when planning to pick up objects (Frak et al., 2001) and when predicting the consequences of one's own and another's actions (Knoblich \& Flach, 2001; Knoblich et al., 2002). However, most of the previous research, although consistent with accounts of simulation, does not give direct evidence for simulation. The interference paradigm developed here may provide one avenue for a more direct test of simulation. If simulation is responsible for planning easy and difficult grasps, then performing a concurrent action when making these judgments should interfere with the effect. Similarly, if simulation is responsible for predicting the outcomes of actions, then performing a concurrent action should interfere with one's ability to predict the outcomes. Studies are currently underway to test these predictions.

One question that remains to be addressed is the neurological locus of the effects of simulation on perception. Single-cell recording studies and imaging experiments specify several possible locations, including the prefrontal, parietal, and supplementary motor cortices and cerebellum (see the introduction, see also Grezes \& Decety, 2001, for review). Once we determine the neurological mechanism supporting simulation, we can begin to explore other processes that are engaged by this mechanism, which could lead to other methods for interfering with simulation that would strengthen our account.

\section{Implications for Theories of Perception}

That information about the perceiver's ability to act influences perception is in conflict with accounts of visual perception that argue that perception is an informationally encapsulated, modular process (e.g., Fodor, 1983; Pylyshyn, 2003). Our results demonstrate that perception integrates information about the external environment with information about the "internal environment" (see Proffitt, 2006) and with information about the perceiver's ability to act. In other words, perception is not just about what is in the environment and, therefore, is not just creating an internal model or a re-presentation of the environment.

Not all perceptual psychologists prescribe to the "internal representation" account of perception. Notably, J. J. Gibson and ecological psychologists were opposed to this notion; instead, they argued that the purpose of perception was not to represent the 
environment but rather to direct action. According to Gibson, this was done by perceiving objects in terms of their affordances, which are the possibilities for action that objects possess (Gibson, 1979). Depending upon its size, shape, and weight, an object may afford grasping, throwing, poking, sitting, and so forth. According to Gibson, affordances exist in the environment and are picked-up by the perceiver based on the perceiver's body and current intentions to act.

We have expanded on Gibson's work to show that perceiving one affordance as opposed to another changes the perceived metric properties of the environment. Because Gibson did not know about these findings prior to his death, we are reluctant to speculate on what he would say about this account. He was, however, adamantly opposed to the idea that perception was augmented by internal knowledge structures and instead argued that perception was direct, meaning that it is influenced only by optical information and not constructed or augmented by cognitive processes. Our results demonstrate that internal information about the perceiver's ability to act influences perception, so perception is influenced by aspects of the perceiver. Therefore, ecological psychologists may be hesitant to accept our claims as they go against the notion of direct perception and, thus, diverge from Gibson in a seemingly fundamental way.

However, the context in which Gibson made his claim for direct perception is quite different than the context in which we claim internal information influences perception. Gibson's argument for direct perception was in response to theories that claimed that internal information was necessary to accurately perceive the environment. Gibson argued that the information available to a moving point of observation was sufficient to adequately specify environmental layout, and, therefore, internal inferences were not necessary. We agree that spatial layout is adequately specified by optical variables. However, it is important to make the distinction between sufficient information for perception and information that is actually incorporated into perception. Just because the optical information is sufficient does not mean that internal information about the perceiver cannot also be informative in perception. Moreover, our reasoning for arguing that internal information about the perceiver's ability to act influences perception resonates with Gibson's entire approach to perception, namely that perception is about affordances.

\section{Embodied Cognition-Embodied Perception}

Increasingly today, there is a sense that a person's body and ability to act need to be included in accounts of perception and cognition. Supporters of this approach have claimed that the body influences mental processes such as language and metaphors (e.g., Lakoff \& Johnson, 1980), categories (e.g., Lakoff, 1987), memory representations (e.g., Glenberg, 1997), the selection of perceptual information (e.g., Ballard, Hayhoe, \& Pelz, 1995), perception of biological motion (e.g., Blake \& Shiffrar, 2007; Wilson \& Knoblich, 2005), and several other cognitive processes (for overviews, see, e.g., Clark, 1997; Wilson, 2002). Each of these cognitive processes has a long history of being investigated in isolation of the body and the context within which it operates; however, new research makes evident the body's significant role in many of the mind's processes.
The current research demonstrates a formative role of the body in spatial perception. In particular, this research shows that when a body's reaching ability is extended, via use of a hand tool, perceived distance is influenced (Witt et al., 2005; current experiments). Moreover, our findings further articulate the embodiedcognition approach by delineating those aspects of the perceiver that have a perceptual influence. Importantly, we have shown that the perceiver's intentions mediate all bodily influences such that only the physiological state and behavioral potential associated with intended actions affects perception (Witt et al., 2004, 2005). Thus, the body, its abilities, and the perceiver's intention influence perception.

As has been demonstrated here, a process underlying the effects of ability and intention on perception is a motor simulation. At present, it is not known whether all embodied influences on perception and cognition share a common underlying mechanism. Certainly motor simulation is poised to inform and influence perception and cognition of a person's current abilities for the intended action. Therefore, embodied effects that are time-specific, such as the selection of perceptual information (Ballard et al., 1995), could take advantage of the outputs of a motor simulation. Research to date, however, does not yet speak to what role, if any, is played by motor simulation in other contexts.

\section{Summary}

In summary, perception is a function of optical information, the perceiver's intention, and the perceiver's ability to perform an intended action. One process that is involved in these effects is an internal, motor simulation. When intending to perform an action, the perceiver simulates the action, and the outcome of that simulation influences perception. When we dissociated between the perceiver's current abilities and simulated abilities, we observed that perception was influenced by the simulated abilities. Furthermore, when we inhibited the simulation such that there was no outcome to inform perception, perceivers did not see the world in terms of their abilities to act. These results suggest that motor simulation is one process through which perception relates the people's behavioral abilities to the affordances of the environment

\section{References}

Ballard, D. H., Hayhoe, M. M., \& Pelz, J. B. (1995). Memory representations in natural tasks. Journal of Cognitive Neuroscience, 7, 66-80.

Bhalla, M., \& Proffitt, D. R. (1999). Visual-motor recalibration in geographical slant perception. Journal of Experimental Psychology: Human Perception and Performance, 25, 1076-1096.

Blake, R., \& Shiffrar, M. (2007). Perception of human motion. Annual Review of Psychology, 58, 47-73.

Chaminade, T., Meary, D., Orliaguet, J. P., \& Decety, J. (2001). Is perceptual anticipation a motor simulation? A PET study. Neuroreport, 12, 3669-3674.

Clark, A. (1997). Being there: Putting brain, body, and world together again. Cambridge, MA: MIT Press.

Decety, J. (1993). Analysis of actual and mental movement times in graphic tasks. Acta Psychologia, 82, 367-372.

Decety, J., \& Grezes, J. (1999). Neural mechanisms subserving the perception of human actions. Trends in Cognitive Sciences, 3, 172-178.

Decety, J., \& Jeannerod, M. (1995). Mentally simulated movements in virtual reality: Does Fitts's law hold in motor imagery? Behavioral Brain Research, 14, 127-134. 
Decety, J., Jeannerod, M., Durozard, D., \& Baverel, G. (1993). Central activation of autonomic effectors during mental simulation of motor actions in man. Journal of Physiology, 461, 549-563.

Decety, J., Jeannerod, M., Germain, M., \& Pastene, J. (1991). Vegetative response during imagined movement is proportional to mental effort. Behavioral Brain Research, 31, 1-5.

Decety, J., Jeannerod, M., \& Prablanc, C. (1989). The timing of mentally represented actions. Behavioural Brain Research, 34, 35-42.

Decety, J., \& Michel, F. (1989). Comparative analysis of actual and mental movement times in two graphic tasks. Brain and Cognition, 11, 87-97.

Decety, J., Perani, D., Jeannerod, M., Bettinardi, V., Tadary, B., Woods, R., et al. (1994). Mapping motor representations with positron emission tomography. Nature, 371, 600-602.

Decety, J., Philippon, B., \& Ingvar, D. H. (1988). rCBF landscapes during motor performance and motor ideation of a graphic gesture. European Archives of Psychiatry and Neurological Sciences, 238, 33-38.

Dominey, P., Decety, J., Broussolle, E., Chazot, G., \& Jeannerod, M. (1995). Motor imagery of a lateralized sequential task is asymmetrically slowed in hemi-Parkinson's patients. Neuropsychologia, 33, 727-741.

Fajen, B. R. (2005). Perceiving possibilities for action: On the necessity of calibration and perceptual learning for the visual guidance of action. Perception, 34, 717-740.

Fitts, P. M. (1954). The information capacity of the human motor system in controlling the amplitude of movement. Journal of Experimental Psychology, 47, 381-391.

Fodor, J. A. (1983). Modularity of mind: An essay on faculty psychology. Cambridge, MA: MIT Press.

Frak, V. G., Paulingnan, Y., \& Jeannerod, M. (2001). Orientation of the opposition axis in mentally simulated grasping. Experimental Brain Research, 136, 120-127.

Gerardin, E., Sirigu, A., Lehericy, S., Poline, J., Gaymard, B., Marsault, C., et al. (2000). Partially overlapping neural networks for real and imagined hand movements. Cerebral Cortex, 10, 1093-1104.

Gibson, J. J. (1979). The ecological approach to visual perception. Boston: Houghton Mifflin.

Glenberg, A. (1997). What memory is for. Behavioral and Brain Sciences, $20,1-55$.

Grezes, J., \& Decety, J. (2001). Functional anatomy of execution, mental simulation, observation, and verb generation of actions: A meta-analysis. Human Brain Mapping, 12, 1-19.

Hanakawa, T., Immisch, I., Toma, K., Dimyan, M. A., van Gelderen, P., \& Hallett, M. (2003). Functional properties of brain areas associated with motor execution and imagery. Journal of Neurophysiology, 89, 9891002.

Ingvar, D. H., \& Philipson, L. (1977). Distribution of cerebral blood flow in the dominant hemisphere during motor ideation and motor performance. Annals of Neurology, 2, 230-237.

Jacobs, A., \& Shiffrar, M. (2005). Walking perception by walking observers. Journal of Experimental Psychology: Human Perception and Performance, 31, 157-169.

Jeannerod, M. (2001). Neural simulation of action: A unifying mechanism for motor cognition. Neuroimage, 14, S103-S109.

Johnson, S. H., Rotte, M., Grafton, S. T., Hinrichs, H., Gazzaniga, M. S., Heinze, H. J. (2002). Selective activation of a parietofrontal circuit during implicitly imagined prehension. Neuroimage, 17, 1693-1704.

Knoblich, G., \& Flach, R. (2001). Predicting the effects of actions: Interactions of perception and action. Psychological Science, 12, 467-472.

Knoblich, G., Seigerschmidt, E., Flach, R., \& Prinz, W. (2002). Authorship effects in the prediction of handwriting strokes: Evidence for action simulation during action perception. The Quarterly Journal of Experimental Psychology, 55A, 1027-1046.

Lakoff, G. (1987). Women, fire, and dangerous things: What categories reveal about the mind. Chicago: University of Chicago Press.
Lakoff, G., \& Johnson, M. (1980). Metaphors we live by. Chicago: University of Chicago Press.

Loomis, J. M., Da Silva, J. A., Fujita, N., \& Fukusima, S. S. (1992). Visual space perception and visually directed action. Journal of Experimental Psychology, 18, 906-921.

Maruff, P., \& Velakoulis, D. (2000). The voluntary control of motor imagery. Imagined movements in individuals with feigned motor impairment and conversion disorder. Neuropsychologia, 38, 1251-1260.

Papaxanthis, C., Pozzo, T., Skoura, X., \& Schieppati, M. (2002). Does order and timing in performance of imagined and actual movements affect the motor imagery process? The duration of walking and writing task. Behavior Brain Research, 134, 209-215.

Papaxanthis, C., Schieppati, M., Gentili, R., \& Pozzo, T. (2002). Imagined and actual arm movements have similar durations when performed under different conditions of direction and mass. Experimental Brain Research, 143, 447-452.

Parsons, L. M. (1987a). Imagined spatial transformation of one's body. Journal of Experimental Psychology: General, 116, 172-191.

Parsons, L. M. (1987b). Imagined spatial transformations of one's hands and feet. Cognitive Psychologie, 19, 178-241.

Parsons, L. M., \& Fox, P. T. (1998). The neural basis of implicit movements used in recognizing hand shape. Cognitive Neuropsychology, 15, 583-615.

Parsons, L. M., Fox, P. T., Downs, J. H., Glass, T., Hirsch, T. B., Martin, C. C., et al. (1995). Use of implicit motor imagery for visual shapediscrimination as revealed by PET. Nature, 375, 54-58.

Petit, L. S., Pegna, A. J., Mayer, E., \& Hauert, C. A. (2003). Representation of anatomical constraints in motor imagery: Mental rotation of a body segment. Brain \& Cognition, 51, 95-101.

Philbeck, J. W., \& Loomis, J. M. (1997). Comparison of two indicators of perceived egocentric distance under full-cue and reduced-cue conditions. Journal of Experimental Psychology, 23, 72-85.

Proffitt, D. R. (2006). Embodied perception and the economy of action. Perspectives on Psychological Science, 1, 110-122.

Proffitt, D. R. (in press). Action-specific perception. In R. B. MacWhinney \& M. Behrmann (Eds.), Embodiment, ego-space, and action: The 34th Carnegie Symposium on Cognition. Hillsdale, NJ: Erlbaum.

Proffitt, D. R., Bhalla, M., Gossweiler, R., \& Midgett, J. (1995). Perceiving geographical slant. Psychonomic Bulletin \& Review, 2, 409-428.

Proffitt, D. R., Stefanucci, J., Banton, T., \& Epstein, W. (2003). The role of effort in distance perception. Psychological Science, 14, 106-112.

Pylyshyn, Z. W. (2003). Seeing and visualizing: It's not what you think. Cambridge, MA: The MIT Press.

Roland, P. E., Skinhoj, E., Lassen, N. A., \& Larsen, B. (1980). Different cortical areas in man in organization of voluntary movements in extrapersonal space. Journal of Neurophysiology, 43, 137-150.

Shepard, R. N., \& Metzler, J. (1971). Mental rotation of three-dimensional objects. Science, 171, 701-703.

Sirigu, A., Cohen, L., Duhamel, J. R., Pillon, B., Dubois, B., Agid, Y., \& Pierrot-Deseilligny, C. (1995). Congruent unilateral impairments for real and imagined hand movements. Neuroreport, 6, 997-1001.

Sirigu, A., Duhamel, J. R., Cohen, L., Pillon, B., Dubois, B., \& Agid, Y. (1996). The mental representation of hand movements after parietal cortex damage. Science, 273, 1564-1568.

Stefanucci, J. K., Proffitt, D. R., Banton, T., \& Epstein, W. (2005). Distances appear different on hills. Perception and Psychophysics, 67, 1052-1060.

Stephan, K. M., Fink, G. R., Passingham, R. E., Silbersweig, A. O., Ceballos-Basumann, A. O., Frith, C. D., \& Frackowiak, R. S. J. (1995). Functional anatomy of the mental representation of upper extremity movements in healthy subjects. Journal of Neurophysiology, 73, 373386.

Wesp, R., Cichello, P., Gracia, E. B., \& Davis, K. (2004). Observing and 
engaging in purposeful actions with objects influences estimates of their size. Perception \& Psychophysics, 66, 1261-1267.

Wexler, M., Kosslyn, S. M., \& Berthoz, A. (1998). Mental processes in mental rotation. Cognition, 68, 77-94.

Wilson, M. (2002). Six views of embodied cognition. Psychonomic Bulletin and Review, 9, 625-636.

Wilson, M., \& Knoblich, G. (2005). The case for motor involvement in perceiving conspecifics. Psychological Bulletin, 131, 460-473.

Witt, J. K., Linkenauger, S. A., Bakdash, J. Z., \& Proffitt, D. R. (in press). Golf performance can make the hole look as big as a bucket or as small as a dime. Psychonomic Bulletin and Review.

Witt, J. K., \& Proffitt, D. R. (2005). See the ball, hit the ball: Apparent ball size is correlated with batting average. Psychological Science, 16, 937-938.

Witt, J. K., Proffitt, D. R., \& Epstein, W. (2004). Perceiving distance: A role of effort and intent. Perception, 33, 577-590.
Witt, J. K., Proffitt, D. R., \& Epstein, W. (2005). Tool use affects perceived distance, but only when you intend to use it. Journal of Experimental Psychology: Human Perception and Performance, 31, 880-888.

Wohlschläger, A., \& Wohlschläger, A. (1998). Mental and manual rotation. Journal of Experimental Psychology: Human Perception and Performance, 24, 397-412.

Wuyam, B., Moosavi, S. H., Decety, J., Adams, L., Lansing, R. W., \& Guz, A. (1995). Imagination of dynamic exercise produced ventilatory responses which were more apparent in competitive sportsmen. Journal of Physiology, 482, 713-724.

Received January 25, 2007

Revision received October 6, 2007

Accepted October 13, 2007

\section{Members of Underrepresented Groups: Reviewers for Journal Manuscripts Wanted}

If you are interested in reviewing manuscripts for APA journals, the APA Publications and Communications Board would like to invite your participation. Manuscript reviewers are vital to the publications process. As a reviewer, you would gain valuable experience in publishing. The P\&C Board is particularly interested in encouraging members of underrepresented groups to participate more in this process.

If you are interested in reviewing manuscripts, please write to the address below. Please note the following important points:

- To be selected as a reviewer, you must have published articles in peer-reviewed journals. The experience of publishing provides a reviewer with the basis for preparing a thorough, objective review.

- To be selected, it is critical to be a regular reader of the five to six empirical journals that are most central to the area or journal for which you would like to review. Current knowledge of recently published research provides a reviewer with the knowledge base to evaluate a new submission within the context of existing research.

- To select the appropriate reviewers for each manuscript, the editor needs detailed information. Please include with your letter your vita. In the letter, please identify which APA journal(s) you are interested in, and describe your area of expertise. Be as specific as possible. For example, "social psychology" is not sufficient-you would need to specify "social cognition" or "attitude change" as well.

- Reviewing a manuscript takes time (1-4 hours per manuscript reviewed). If you are selected to review a manuscript, be prepared to invest the necessary time to evaluate the manuscript thoroughly.

Write to Journals Office, American Psychological Association, 750 First Street, NE, Washington, DC 20002-4242. 